\title{
Approches méthodologiques de la sécurité : engagements, obstacles et défis
}

Introduction

Emmanuel-Pierre Guittet

\section{OpenEdition}

\section{Journals}

Édition électronique

URL : http://journals.openedition.org/conflits/19262

DOI : $10.4000 /$ conflits. 19262

ISSN : 1777-5345

Éditeur :

CCLS - Centre d'études sur les conflits lilberté et sécurité, L'Harmattan

Édition imprimée

Date de publication : 8 août 2016

Pagination : 7-15

ISBN : 978-2-343-10075-3

ISSN : 1157-996X

\section{Référence électronique}

Emmanuel-Pierre Guittet, «Approches méthodologiques de la sécurité : engagements, obstacles et défis », Cultures \& Conflits [En ligne], 102 | été 2016, mis en ligne le 08 août 2016, consulté le 30 mars 2021. URL : http://journals.openedition.org/conflits/19262 ; DOI : https://doi.org/10.4000/conflits. 19262 


\title{
Approches méthodologiques de la sécurité : engagements, obstacles et défis
}

\author{
Introduction
}

\section{Emmanuel-Pierre GUITTET}

Chercheur associé an Centre d'Études sur les Conflits, Liberté et Sécurité (CCLS, France), directeur de la collection New Approaches to Conflict Analysis aux Presses de l'Université de Manchester et co-directeur de la collection Routledge Studies in International Political Sociology.

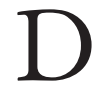

epuis leur apparition à l'orée des années 1990, les études critiques de sécurité n'ont eu de cesse de gagner du terrain. Érodant progressivement les conceptions traditionnelles des Relations Internationales, elles ont contribué à remettre en cause certains poncifs de la discipline et concouru à l'élargissement du champ d'investigation de la sécurité au-delà de la seule dimension militaire ${ }^{1}$. En mettant l'accent sur la multiplicité des acteurs prétendant dire et faire la sécurité, les études critiques ont déstabilisé l'ossature stato-centrée des Relations Internationales et ont souligné la fluidité même du terme de sécurité et, par conséquent, de ses usages politiques et effets sociaux, permettant ainsi de décloisonner un objet d'étude resté jusque-là l'apanage des seules Relations Internationales ${ }^{2}$.

En ouvrant l'horizon du questionnement, les études critiques ont également amélioré la compréhension des conditions de production de la connaissance savante et ordinaire, des jeux d'expertise et des discours d'autorité sur la sécurité. L'intérêt porté à la sédimentation des discours, des luttes de pouvoir, des échanges et des jeux de concurrence en la matière a produit un ensemble de recherches pertinentes, à même de redéfinir la question posée : non pas dis-

1. Buzan B., Waever O., de Wilde J., Security: A New Framework For Analysis, Boulder, Lynne Rienner, 1998.

2. Macleod A. (dir.), "Approches critiques de la sécurité : une perspective canadienne ", Cultures E Conflits, n ${ }^{\circ}$ 54, 2004 ; Gilles B., Delori M., "Études critiques de sécurité. Introduction ", Etudes internationales, vol. XLVI, n² 2-3, 2015, pp. 139-145. 
cuter ce qu'est la sécurité mais s'attacher à comprendre qui dit et que fait la sécurité. Plus récemment, avec l'introduction de recherches sur les outils de la sécurité et de la surveillance soumis au rythme du principe de précaution et de la mise en mots du risque et de leurs conséquences en termes de liberté, d'obéissance et de gestion des peurs, c'est toute une réflexion extrêmement riche sur les capacités technologiques et bureaucratiques à structurer les relations sociales et politiques qui s'est ouverte et dont la revue Cultures $\mathcal{E}$ Conflits, entre autres, s'est fait le porte-voix ${ }^{3}$.

Qu'elles soient abordées en termes d'écoles ${ }^{4}$, de champs d'étude ou encore de théories complémentaires ou concurrentes, les études critiques de sécurité sont aussi riches que variées. La richesse des travaux entrepris n'est pas sans refléter l'extension même des domaines d'action de la sécurité, le développement sans précèdent d'une véritable industrie éponyme ${ }^{5}$ et la reformulation des frontières entre privé et public ${ }^{6}$. Une recherche aussi qui s'efforce de rendre compte de la constitution d'un « ordre digital » des pratiques 7 , des savoirs, des codifications et des discours de vérité de la sécurité ${ }^{8}$. Et finalement, toute une recherche qui s'emploie à analyser la concentration, la multiplication et la mobilité des acteurs produisant des discours et des effets visi-

3. Bigo D., «Le nexus sécurité, frontière, immigration : programme et diagramme », Cultures $\varepsilon$ Conflits, $n^{\circ}$ 84, 2011, pp. 7-12 ; Bigo D., Piazza P. (dir.), «Fichage et listing. Quelles incidences pour les individus ? ", Cultures E Conflits, n 76, 2009 ; Bigo D. (dir.), " Sécurité et protection des données ", Cultures $\mathcal{E}$ Conflits, $n^{\circ}$ 74, 2009.

4. Waever O., "Aberystwyth, Paris, Copenhagen: New "Schools" in Security Theory and their Origins between Core and Periphery », Annual Convention of the International Studies Association, Montreal, 17-20 Mars 2004 ; Van Munster R., « Security on a Shoestring: A Hitchiker's Guide to Critical Schools of Security in Europe », Cooperation and Conflict, vol. 42, $\mathrm{n}^{\circ}$ 2, 2007, pp. 235-243.

5. Hoijtink M., "Capitalizing on emergence: The "new” civil security market in Europe ", Security Dialogue, vol. 45, n 5, 2014, pp. 458-475 ; Leander A. (dir.), The Commercialization of Security in Europe: Consequences for Peace and Reconciliation, New York, Routledge, 2013 ; O’Reilly C., " The transnational security consultancy industry. A case of state-corporate symbiosis ", Theoretical Criminology, vol. 14, n² 2, 2010, pp. 183-210 ; Guittet E.-P., Jeandesboz J., "Security technologies ", in Burgess J.P. (dir.), The Routledge Handbook of New Security Studies, Londres, Routledge, 2010, pp. 229-239.

6. Lorenc Valcarce F., La sécurité privée en Argentine. Entre surveillance et Marché, Paris, Editions Karthala, 2011 ; Wakefield A., Selling security: the private policing of public space, Devon, Willan Publishing, 2003 ; De Waard J., "The private security industry in international perspective ", European journal on criminal policy and research, vol. 7, n 2, pp. 143-174, 1999 ; Ocqueteau F., Pottier M.-L., Warfman D., «La sécurité privée en France », Les Cabiers de la sécurité intérieure, ${ }^{\circ} 33,1998$, pp. 105-127 ; Lemaitre A., « Le rôle de l'industrie de l'assurance dans le pilotage de la sécurité privée et de la politique de prévention ", Déviance et société, vol. 19, $\mathrm{n}^{\circ} 2,1995$, pp. 159-168.

7. Ruppert E., Law J., Savage M., « Reassembling Social Science Methods: The Challenge of Digital Devices ", Theory, Culture E Society, vol. 30, n 4, 2013, pp. 22-46 ; Amoore L., «Data Derivatives. On the Emergence of a Security Risk Calculus for Our Times », Theory, Culture E Society, vol. 28, n 6, 2011, pp. 24-43.

8. Huysmans J., Security Unbound. Enacting Democratic Limits, Abingdon, Routledge, 2014. Bonditti P., «Les concepts, parent pauvre des études (critiques) de sécurité ? Proposition pour une archéologie des savoirs de la sécurité ", Études Internationales, vol. XLVI, n 2-3, 2015, pp. 167-188 ; Hansen L., Security as Practice: Discourse Analysis and the Bosnian War, Londres, Routledge, 2006. 
bles de sécurité autant que des effets d'invisibilisation de ses conséquences. La variété de ces études se traduit par des approches théoriques construites autour de postulats philosophiques et de sensibilités politiques différents, fruits à la fois de traditions et de contextes nationaux mais aussi structurées par des positionnements disciplinaires et institutionnels des chercheurs évoluant au sein de ces études.

Les controverses nées autour de la division en trois grandes écoles se disputant la paternité de la critique continuent d'alimenter des discussions acrimonieuses et par trop souvent stériles au sein des petites arènes académiques 9 . Comme le suggèrent Gilles Bertrand et Mathias Delori dans un numéro spécial de la revue Études Internationales consacré aux études critiques de la sécurité, il est fort probable que ces disputes scholastiques aient terni la portée même et les possibilités du projet critique originel ${ }^{10}$.

En revanche, il nous parait plus hasardeux d'en tirer la conclusion que les études critiques de sécurité s'essoufflent pour autant. Les publications brandissant fièrement les termes de critique et de sécurité semblent être moins nombreuses certes mais il serait contre les bons usages de proclamer la mort $\mathrm{du}$ roi sans avoir vérifié son trépas. Premièrement, nombreux sont ceux qui ont délaissé ces batailles sémantiques de salon pour y préférer les questions pluridisciplinaires et les enjeux politiques, en offrant des analyses crédibles et concrètes des pratiques de sécurité et des politiques d'insécurité qui contribuent à la reconfiguration inquiétante de nos espaces politiques ${ }^{11}$. Deuxièmement, il conviendrait aussi de se pencher sur le changement d'échelle au sein des études de sécurité avec le développement d'un intérêt beaucoup plus marqué pour la matérialité 12 et les effets ordinaires, contraignants et structurants des pratiques de sécurité auprès des acteurs de sécurité ${ }^{13}$ et du plus grand public ${ }^{14}$. Une inflexion des recherches au sein des

9. Voir à cet égard le manifeste du Collectif CASE et les réponses qu'il a suscitées, mais aussi la tentative plus récente d'initier une école canadienne d'études critiques de la sécurité : C.A.S.E. Collective, "Critical Approaches to Security in Europe. A Networked Manifesto », Security Dialogue, vol. 37, n 4, 2006, pp. 443-487 ; Behnke A., "Presence and creation: A few (meta-)critical comments on the CASE manifesto ", Security Dialogue, vol. 38, n 1, 2007, pp. 105-111. Sur l'école canadienne de sécurité, voir de Larrinaga M., Salter M. B., " Cold CASE: a manifesto for Canadian critical security studies ", Critical Studies on Security, vol. 2, $\mathrm{n}^{\circ}$ 1, 2014, pp. 1-19. Sur le Cold CASE, voir entres autres les réponses d'Alex Macleod et de Peter Stoett dans le même numéro : Macleod A., "Cold case: some thoughts on the sociology of Canadian critical security studies », Critical Studies on Security, vol 2, n 1, 2014, pp. 2630 ; Stoett P., "Critical reflections on de Larrinaga and Salter », Critical Studies on Security, vol. $2, n^{\circ} 1,2014$, pp. 23-25.

10. Bertrand G., Delori M., op. cit., p. 141.

11. Basaran T., Bigo D. Guittet E.-P., Walker R.B.J. (dir), International Political Sociology. Transversal Lines, Londres, Routledge, 2016 ; Bigo D., Guittet E.-P., Scherrer A. (dir.), Mobilité(s) sous surveillance. Perspectives croisées UE - Canada, Montréal, Athéna, 2010; Huysmans J., The Politics of Insecurity. Fear, Migration and Asylum in the EU, Londres, Routledge, 2006.

12. Enloe C., "The Mundane Matters ", International Political Sociology, n 5, 2011, pp. 447-50.

13. Bonelli L., Ragazzi F., « Low-tech security: Files, notes, and memos as technologies of antici- 
études critiques de sécurité qui procède de la multiplication heureuse de travaux de terrain plus approfondis. Les termes des débats se sont complexifiés et il serait dès lors important de prendre la mesure de l'impact des études sur les risques, du renouvellement des discussion sur les formes et les limites de la modernité ainsi que les allers-retours enrichissants entre les problématiques de sécurité et le développement sans précédent de pratiques de surveillance 15 avant d'annoncer « l'essoufflement » des études critiques de sécurité. La portée critique et innovante des études de sécurité s'énonce au pluriel de nouveaux questionnements. C'est d'ailleurs l'un des enjeux important mis en exergue par Christophe Wasinski et Christian Olsson dans leurs contributions au numéro spécial de la revue Études Internationales 16 ; loin d'être finies, les études critiques de sécurité ont, au contraire, contribué à ouvrir de nouveaux dialogues transdisciplinaires qui ne demandent qu'à être poursuivis et affinés.

Si depuis les années 1990, les études critiques de sécurité ont progressivement gagné du terrain, suscitant à la fois curiosité, intérêt mais aussi parfois dédain, elles n'ont pas échappé au regain d'intérêt pour la méthodologie qui a déferlé ces dernières années sur les Relations Internationales en particulier, et les sciences sociales en général. Nées du «tournant constructiviste » en Relations Internationales initié dans les années 1980, serait-il possible que les études critiques de sécurité soient désormais au pied du mur et sommées de répondre à ce nouveau «tournant méthodologique » ? La question de déterminer ce qui fait et comment se fait une approche critique de la sécurité n'est pas dénuée de sens et a été régulièrement abordée sous l'angle du rapport à la réflexivité ${ }^{17}$. Pédagogiquement parlant, il s'agit là d'une question qui revient souvent chez les étudiants curieux et avides d'appareillages critiques mais néanmoins anxieux quant aux procédés. Au sein de cette diversité d'objets et d'approches théoriques, existe-t-il une méthodologie en particulier répondant aux impératifs critiques de ces études ?

pation », Security Dialogue, vol. 45, n 5, 2014, pp. 476-493 ; Goold B., Loader I., Thumala A., « The Banality of Security. The Curious Case of Surveillance Cameras », British Journal of Criminology, vol. 53, $\mathrm{n}^{\circ}$ 6, 2013, pp. 977-996 ; Salter M.B., Politics at the Airport, Minneapolis et Londres, University of Minnesota Press, 2008.

14. Jarvis L., ListerM., Anti-terrorism, citizenship and security, Manchester, Manchester University Press, 2015.

15. Bauman Z., Bigo D., Esteves P., Guild E., Jabri V., Lyon D. et R. B. J. Walker, « After Snowden: Rethinking the impact of surveillance ", International political sociology, vol. 8, $\mathrm{n}^{\circ}$ 2, 2014, pp. 121-144 ; Amoore L., The Politics of Possibility: Risk and Security beyond Probability, Durham, Duke University Press, 2013.

16. Sur la possibilité et la nécessité d'ouvrir un dialogue avec les études militaires critiques, voir Wasinski C., "Les sciences humaines et la critique de la raison stratégique » et Olsson C., "De la sécurité à la violence organisée : tropismes et points aveugles de "l’École de Copenhague” », in Bertrand G., Delori M., op. cit., pp. 211-230.

17. Bigo D., "Grands débats dans un petit monde », Cultures E Conflits, n 19/20, 1995, pp. 748 ; Huysmans J., « Dire et écrire la sécurité : le dilemme normatif des études de sécurité », Cultures E Conflits, $\mathrm{n}^{\circ}$ 31-32, 1998, pp. 177-202 ; Huysmans J., « Defining Social Constructivism in Security Studies: The Normative Dilemma of Writing Security », Alternatives: global, local, political, $\mathrm{n}^{\circ} 27$ (Special issue), 2002, pp. 41-62. 
Depuis quelques années déjà, la question fait l'objet d'un nombre grandissant de publications en langue anglaise 18 et la notion de rigueur scientifique semble faire un retour triomphant ${ }^{19}$. Il serait tentant de lire ce phénomène comme le résultat d'un retour en grâce de ce que Karl Popper nommait à juste titre la « rengaine ennuyeuse » entre sciences dures et sciences humaines, entre des sciences fortes de méthodes et d'autres supposées dépourvues de tels outils ${ }^{20}$. Au-delà de ce sempiternel débat autour de la scientificité comme exercice de distinction académique, l'explication de cet intérêt grandissant pour les méthodes et les méthodologies au sein des études critiques de sécurité pourrait bien être beaucoup plus prosaïque; et si les études critiques de sécurité étaient victimes de leur succès ? La prétention à l'assise institutionnelle n'est plus une quête mais une réalité et les études critiques de sécurité sont désormais sommées de présenter leurs lettres de noblesse scientifique. Néanmoins, cet appel à la rigueur et à la clarté méthodologique au sein des études critiques de sécurité ${ }^{21}$ suggère une absence initiale, une tare essentielle entachant le sérieux de l'entreprise et qu'il conviendrait de corriger. Dans un article récent sur les approches ethnographiques au sein des études de sécurité, Anna Leander rappelle d'ailleurs que d'un point de vue extérieur, le consensus semble être que ces études sont méthodologiquement « sous-développées, évasives et confuses 22 ». Anna Leander ne partage pas ce scepticisme de bon aloi et souligne à bon escient d'ailleurs qu'il s'agit d'une image déformée des études critiques de sécurité, au sein desquelles les questions relatives à l'objectivité et à l'exercice de neutralité épistémologique sont bien plus développées que ses détracteurs veulent bien l'admettre. En revanche, les défis méthodologiques restent entiers.

Les réponses varient certes, mais n'en demeurent pas moins enfermées dans une vision somme toute très classique de ce que peut être la méthodologie (une méthodologie), de ce que peut être la théorie (une théorie) et, finalement de ce que peut être une critique (la critique). Ces aspects du travail scientifique ont souvent été pensés de manière exclusive, orthodoxe et séparés les uns des autres comme le suggère le titre et la composition du volume dirigé par Laura Sheperd 23 : « une introduction aux théories et aux méthodes ». A

18. Voir entres autres Aradau C. et al., Critical Security Methods. New Frameworks for Analysis, Londres, Routledge, 2014 ; Salter M. et al., Research Methods in Critical Security Studies. An Introduction, Londres, Routledge, 2013 ; Shepherd L. (dir.), Critical Approaches to Security. An Introduction to Theories and Methods, Londres, Routledge, 2013.

19. Yanow D., « Neither rigorous nor objective? Interrogating criteria for knowledge claims in interpretive science ", in Yanow D., Schwartz-Shea P. (dir.), Interpretation and Method: Empirical Research Methods and the Interpretive Turn, New York, M.E. Sharpe, 2006, pp. 67-88.

20. Popper K., La connaissance objective, Paris, Aubier, 1972, p. 287.

21. L'ouvrage coordonné par Mark Salter et Can E. Mutlu (op. cit.) est un appel sans équivoque possible. L'ouvrage se présente comme " a desire to champion clear research design and rigorous method in critical security studies " (introduction, p. 1).

22. Leander A., "Ethnographic Contributions to Method Development: "Strong Objectivity" ", Security Studies, International Studies Perspectives, n 0, 2015, p. 2.

23. Shepherd L. (dir.), op. cit., 2013. 
contrario, l'ouvrage plus récent coordonné par Claudia Aradau, Jef Huysmans, Andrew Neal et Nadine Voelkner met un point d'honneur à réfuter cette logique de séparation entre méthodes, théories et pratiques de recherche et accepte les mots et le choix d'une posture certes imparfaite mais toujours en mouvement ${ }^{24}$. Il ne s'agit pas d'un simple exercice de circulation entre des termes mais bel et bien une position visant à l'enrichissement des pratiques de recherche.

Ce numéro de Cultures $\mathcal{E}$ Conflits s’inscrit dans cette démarche intellectuelle réflexive qui a toujours été au cour de la revue ${ }^{25}$. Il ne s'agit donc pas de produire un exposé général des méthodes disponibles mais bel et bien d'aborder les méthodes à partir non seulement des dispositifs théoriques mis en place, mais aussi et surtout en dialogue avec des recherches, des matériaux et des terrains d'investigation qui mettent la théorie et la méthode à l'épreuve ${ }^{26}$. La méthode ne précède pas l'objet pour autant que l'objet d'étude, la méthode et les sources employées sont intrinsèquement liés. En s'écartant résolument de toute forme d'idolâtrie méthodologique, ce numéro vise à ouvrir un espace de dialogue sur les pratiques méthodologiques à l'épreuve du réel et les «bricolages méthodologiques » comme source, obstacle et espace pour le développement d'une critique affinée, assurée et engagée au sein des études de sécurité. Que l'on parle "d'attention oblique 27 », de «braconnage 28 »ou de «bricolage » comme forme de débrouillardise ${ }^{29}$ ou que l'on parle de métis ${ }^{30}$, il s'agit ici d'intégrer la nécessité de penser l'ajustement (tekhné), la variation et l'inattendu mais aussi le processus créatif en soi.

Ce numéro entend être un plaidoyer pour la flexibilité et la curiosité comme outils de l'analyse. Il ne s'agit pas d'un plaidoyer pour une interprétation libre - ou libérée - faite essentiellement d'intuitions littéraires et de commentaires de texte, ni une forme d'errance plus ou moins nonchalante et sousestimant la dimension historique de l'objet considéré. Mais bel et bien une curiosité et une flexibilité comme mouvement sceptique ou vigilance épistémologique au cœur même de la démarche des sciences sociales qui ne s'opère bien souvent que par parenté et par écart ou, selon l'expression de JeanClaude Passeron, par l'exercice de véridicité ${ }^{31}$. Car ne s'agit-il pas «d'allon-

24. Aradau C. et al., op.cit., 2014.

25. En ce sens, ce numéro renoue le fil d'une discussion entamée dans un précèdent numéro établi sous la direction de Valérie Amiraux et de Daniel Cefai. Voir Amiraux V., Cefai D. (dir), "Les risques du métier. Engagements problématiques en sciences sociales », Cultures $\varepsilon$ Conflits, $n^{\circ} 47$, automne 2002.

26. Hamidi C., « De quoi un cas est-il le cas ? ", Politix, n 4, 2012, pp. 85-98.

27. Hoggart R., La culture du pauvre : étude sur le style de vie des classes populaires en Angleterre, Paris, Éditions de Minuit, 1970.

28. De Certeau M., L'invention du quotidien, Paris, Gallimard, 1990.

29. Sur la notion de bricolage, voir Odin F., Thuderoz C., Des mondes bricolés? Arts et sciences à l'épreuve de la notion de bricolage, Lausanne, PPUR Presses polytechniques, 2010.

30. Detienne M., Vernant J.-P., Les ruses de l'intelligence : La Métis des grecs, Paris, Flammarion, 1974. 
ger le questionnaire ", de déplacer son regard et de produire ainsi une organisation renouvelée de l'observation empirique 32 ? Penser, travailler et rendre compte de manière critique de la multiplicité, de la complexité et de l'imprévu analytique de la sécurité passe inévitablement par l'appropriation et la discussion de ces considérations méthodologiques et épistémologiques. Les articles de ce numéro de la revue Cultures $\mathcal{E}$ Conflits sont construits autour des expériences de terrain de leurs auteurs, de leurs démarches intellectuelles, des outils d'exploitation de données et des techniques et dispositifs théoriques mis en place puis renégociés en fonction des avancées et des difficultés rencontrées, mais aussi des dynamiques spatiales et temporelles propres au terrain observé.

Faisant écho au précédent numéro de la revue Cultures $\varepsilon$ Conflits sur les études critiques de sécurité dont il avait la direction ${ }^{33}$, Alex MacLeod offre ici ses réflexions sur les avantages d'une lecture attentive de la culture populaire pour interroger les paradigmes sécuritaires d'une part et renouveler la critique d'autre part. Dans son article intitulé «La culture populaire : un espace à explorer pour les études critiques de sécurité »Alex MacLeod expose ainsi comment une méthode qualitative et interprétative critique peut se faire et ce qu'elle peut produire en terme de renouveau afin d'affiner les paradigmes théoriques. Julien Jeandesboz, quant à lui, dans son article «Terrain vertueux, engagements problématiques : la vie sociale des méthodes et la recherche sur les questions de sécurité en milieu européen ", nous ouvre ses carnets de recherche et nous livre ses réflexions sur son expérience de chercheur arpentant les couloirs des institutions européennes à la fois à la rencontre des acteurs de la sécurité européenne, mais aussi en tant que chercheur-expert « embarqué »dans la définition des politiques publiques européennes en matière de sécurité. Julien Jeandesboz expose les difficultés de sa position ambivalente de chercheur à la fois «engagé » et "distancié » et contribue ainsi au débat anglo-saxon sur la «vie sociale des méthodes » (Social Life of Methods). Dans leur article intitulé «Analyse du champ de la sécurité en Suisse : émergence des Schengen Boys et autres réflexions méthodologiques », Stephan Davidshofer, Jonas Hagmann et Amal Tawfik nous livrent une plongée dans les arcanes complexes et par trop souvent méconnues des acteurs et des institutions de la sécurité helvétique. Fruit d'une recherche en cours mais néanmoins d'ores et déjà nourrie d'une impressionnante collection d'entretiens et de questionnaires, cet article apporte non seulement une nouvelle pierre à l'hypothèse du champ de la sécurité mais aussi expose pas à pas comment l'analyse des correspondances multiples, l'analyse en composantes prin-

31. Passeron J.-C., Le raisonnement sociologique: l'espace non-poppérien $d u$ raisonnement naturel, Paris, Nathan, 1991, et du même auteur, "L'espace mental de l'enquête (I). La transformation de l'information sur le monde dans les sciences sociales ", Enquête. Archives de la revue Enquête, $\mathrm{n}^{\circ} 1,1995$, p. 13-42.

32. Veyne P., Comment on écrit l'bistoire, Paris, Seuil, 1975.

33. Macleod A. (dir.), op. cit., 2004. 
cipales et l'analyse de réseaux peuvent être mobilisées et travaillées dans ce contexte helvétique. Dans son article « La gouvernance polycentrique du cybercrime : Les réseaux fragmentés de la coopération internationale », Benoit Dupont explore la dimension polycentrique de la gouvernance du cybercrime au moyen notamment des outils de l'analyse de réseaux (réseaux 2-mode). Benoit Dupont montre ainsi combien l'argument du retard de la coopération policière sur les réseaux criminels ne tient pas, bien au contraire. La coopération internationale s'est adaptée à l'émergence d'Internet et si cette gouvernance du crime reste fragmentée, elle demeure somme toute assez hiérarchisée. Au-delà de ces conclusions, Benoit Dupont montre comment une analyse de réseaux s'élabore, se travaille et se redéfinit en fonction des enjeux théoriques et des obstacles méthodologiques. Dans «Les élites européennes comme champ(s)», Frédéric Lebaron propose un retour réflexif sur les usages de la prosopographie et de l'analyse géométrique des données à partir de trois expériences de recherche collective sur l'évolution et les structurations des élites européennes. Frederic Lebaron expose un certain nombre de résultats tangibles mais sans pour autant dissimuler les difficultés inhérentes à de tels projets de recherche collective qui visent à croiser des démarches qualitatives et quantitatives afin de restituer la complexité et l'enchevêtrement de parcours biographiques et professionnels. Dans sa chronique bibliographique «Aux prises avec les vibrations du social : retour sur le practice turn en relations internationales ", Julien Pomarède propose une lecture assidue, sérieuse et sériée de quatre ouvrages récents qui se font fort d'exposer, penser et renouveler l'analyse des pratiques en relations internationales. À la suite de la contribution de Frédéric Lebaron, il souligne combien la tentation de réifier les entités collectives est un enjeu théorique et méthodologique fort qu'il convient de prendre à bras le corps. Julien Pomarède souligne aussi combien ce retour méthodologique sur les pratiques au sein des Relations Internationales ne résout pas à lui seul les questions d'échelle de ce qu'international veut dire, pas plus qu'il ne renferme la clef ultime de la compréhension de l'agir humain.

Ce numéro est aussi l'occasion d'accueillir un article en hors-thème portant sur les mobilisations politiques de la diaspora arménienne, "Quand les Apatrides et les Infidèles contestent : territoires, conflits, innovations ». Pinar Selek y expose les résultats de deux années d'enquête et de recueil de témoignages dans plusieurs villes françaises ainsi qu'en Belgique, en Italie, en Suisse, en Turquie et en Arménie. En revenant pas à pas sur l'histoire et les histoires de ces communautés arméniennes multiples, Pinar Selek montre comment l'expérience puis la mémoire de l'horreur du génocide, du déracinement, de l'exil et de la survie ont structuré progressivement l'identité de la diaspora arménienne. Elle met aussi en lumière la complexité des relations parfois tendues entre les arméniens en exil et les arméniens demeurés en Turquie. Au cœur de cet article, c'est la question des entrelacements de différents espaces 
et de ce que cela peut produire en terme de mobilisation et de contestation, entre un ici et un là-bas, y compris un là-bas imaginaire, rêvé ou espéré. En reprenant les cadres et les termes de la sociologie des mobilisations, Pinar Selek nous invite à repenser comment les mobilisations autour de la question arménienne ont évolué en fonction des opportunités et des restrictions imposées par les relations internationales et les possibilités au local.

La conclusion de Pinar Selek fait parfaitement écho à l'ensemble des contributions de ce numéro : étudier et rendre compte de la complexité des opérateurs, des opérations et des effets de sécurité suppose de prendre en compte des espaces, des durées, des individus et des expériences individuelles et collectives qui évoluent de manière simultanée, mais pas forcément de manière synchronisée. Savoir rendre compte des coïncidences et des concomitances, autant que des ruptures et des dispersions est un défi théorique, méthodologique et épistémologique de taille qui n'est pas propre aux seules études de sécurité. Ce numéro de la revue Cultures $\mathcal{E}$ Conflits souhaite apporter des pistes de réflexion et susciter des discussions. 